\title{
On the Model Containing Coupled Subsystems
}

\author{
Valentin N. Tkhai, Ivan N. Barabanov
}

\begin{abstract}
The model containing coupled subsystems (MCCS), which decouples into independent subsystems as the coupling vanishes, is considered. The subsystems of the MCCS are described by autonomous systems of ordinary differential equations. Each subsystem is supposed to admit a single parameter family of periodic motions. The general characteristics of the MCCS are presented. Some problems concerning the MCCS are stated, i.e. the problems of oscillations existence, bifurcations, stability, stabilization, and resonance. The classification of MCCS is proposed, the currently investigated classes of MCCS are announced. One class of MCCS is studied in detail, the corresponding results are presented.
\end{abstract}

\section{Introduction}

In this paper we study the model containing coupled subsystems. This model is described by a system of ordinary differential equations (ODE), where subsystems are autonomous ODEs. The coupling is characterized by a numeric parameter $\varepsilon$, which can be either scalar or vector. The subsystems become independent as $\varepsilon=0$. If $\varepsilon$ is vector then the MCCS can be of hierarchical structure, where subsystems are coupled on various levels. In the general case subsystems are either linear or nonlinear, of arbitrary order, and of various nature. The $N$-planet problem serves as an example of the MCCS with single-leveled subsystems. The following are some more examples of the MCCS: multi-link pendulum, chain of spring oscillators, Sun-planets-satellites system (a system of two levels), translationally and rotationally moving space vehicles, in particular, artificial Earth satellites, robot systems with cross-coupling, model of DNA oscillations, coupled neurons, wind turbine (electromechanical system), mechatronic systems, etc.

\section{The Model containing Coupled Subsystems (MCCS)}

The concept of MCCS emerged as a result of the formalization of applied problems with mathematical models given by ODEs. This concept can also be implied for systems with

\footnotetext{
Manuscript received March 23, 2014; accepted July 27, 2014.

Valentin N. Tkhai and Ivan N. Barabanov are with the Trapeznikov Institute of Control Sciences, Moscow, Russia.
} 
distributed parameters (for example, for the problem of induction heating), as well as for discrete-time problems. Earlier subsystems were studied separately, whereas the concept of MCCS allows to take into account the coupling subsystems. The models of subsystems appear to be self-sufficient, hence, the coupling of subsystems, on the one hand, must be weak. However, when the interactions between the subsystems are modeled by forces, the forces can be large enough in the general case (example: mathematical pendulum with oscillating pivot).

The concept of MCCS was introduced in [1]. Among the characteristic features of the MCCS there are: multi-level hierarchical structure, various modes of functioning, nonlinearity, high order. MCCS also can be classified as large-scale system. We can consider either autonomous or non-autonomous MCCS.

Concerning MCCS, it is obvious that a) MCCS actually exist, regardless of the way we choose to study them; b) we need to classify MCCS with respect to their structure; c) coupling in MCCS must be considered according to the 3rd Newton's law, i.e. action is equal to reaction; d) MCCS naturally decouples into independent subsystems as the coupling vanishes.

The natural approach to study the dynamics. The structure of the MCCS makes us to imply an approach, which can be called natural. This approach assumes the following: classification of subsystems according to their dynamical properties; analysis of typical links of subsystems.

Unlike previous approaches of decoupling proposed by Matrosov [2-4], Zubov [5], Merkin [6], Pyatnitskiy [7], Siljak [8], Chernousko et al. [9], the current approach essentially takes into account the structure of MCCS, and considers coupling according the principle "action is equal to reaction".

Oscillations, Bifurcations, Stability, Stabilization, Resonance. Basing on the natural approach let us give the general statement of the problems of oscillations, bifurcations, stability, stabilization, and resonance: find conditions for oscillations to exist, describe bifurcation scenarios of oscillations, find stability conditions for oscillations in MCCS, find stabilization conditions in terms of coupling controls, analyze resonance effects in MCCS. This approach implies the analysis of the structure of MCCS, which results in some problem statements depending on subsystem dynamics. Previously obtained results for subsystems contribute to the problem of MCCS.

Classes of MCCS. We study single-frequency oscillations, i.e. periodic solutions. Since subsystems are autonomous, we have isochronous single-frequency oscillations in the linear model, while in the nonlinear model in the non-degenerate case there is an alternative: either a cycle or a family of periodic solutions with the period depending on a single parameter [10] is realized (i.e. the law [11-13] holds).

Now some results are obtained for the following classes of MCCS: autonomous MCCS in the case of family [10] $(A)$; periodic MCCS in the case of family [1] $(B)$; autonomous MCCS in the case of cycle [14] (C); periodic MCCS in the case of cycle [14] $(D)$. MCCS described by reversible mechanical systems [15] (subclasses $A_{A}, A_{B}$ ) are also investigated. Some results are published, others are in print. 
The following definition is used to classify modes of MCCS in the case of family. Consider an autonomous subsystem, where the period is the function of the numerical parameter $T(h)$.

Definition 1. The point of the family of oscillations is called ordinary (o-point) if $d T / d h \neq$ 0 at this point, otherwise the point is called critical (c-point). A critical point can degenerate into an equilibrium (e-point).

We will call o-mode, c-mode, and e-mode the modes of oscillations in subsystems if they correspond to o-point, c-point, and e-point, respectively.

For a single-leveled MCCS there are qualitatively different combinations of modes:

$$
\begin{aligned}
& o-o, \quad o-c, \quad o-e, \quad c-c, \quad c-e, \quad e-e, \quad o-o-c, \quad o-c-c \\
& o-o-e, \quad o-e-e, \quad c-c-e, \quad c-e-e
\end{aligned}
$$

Although there are many combinations, only two or three of them will be of importance. This is due to the law [11-13]. According to this law, the o-point is a rule for a family. Hence, we need to study the combinations containing o-points first. Let us call the mode containg only o-points the main oscillations mode of MCCS.

We consider below MCCS of classes $A$ and $A_{B}$ in the main oscillations mode.

\section{MCCS of class A}

Consider a smooth single-leveled MCCS with $m$ subsystems:

$$
\dot{x}^{s}=X^{s}\left(x^{s}\right)+\varepsilon \tilde{X}^{s}\left(\varepsilon, x^{1}, \ldots, x^{m}\right), \quad x^{s} \in R^{m_{s}}, \quad s=1, \ldots, m, \quad \Sigma m_{s}=n .
$$

Each $s$-th subsystem $(s=1,2, \ldots, m)$ is supposed to admit the family of periodic motions:

$$
x^{s}=\varphi^{s}\left(h_{s}, t+\gamma_{s}\right),
$$

the period $T_{s}$ depending on $h_{s}$. The variables $\gamma_{s}$ in (2) represent the shift along the trajectory of the autonomous system. Then the generating system (i.e. (1) at $\varepsilon=0$ ) admit a family of conditionally periodic solutions with $m$ frequencies. Suppose that there are $2 \pi$-periodic solutions among them. Now we are to find if (1) admits a periodic motion at sufficiently small $\varepsilon \neq 0$ such that this periodic solution tends to the $2 \pi$-periodic solution of the generating system as $\varepsilon \rightarrow 0$.

Denote $h^{*}=\left(h_{1}^{*}, \ldots, h_{m}^{*}\right)$ the vector of parameters that corresponds to the $2 \pi$-periodic solution of the generating system. So we need to find a vector $h^{*}$ such that the perturbations satisfy the conditions of existence of periodic solution at $\varepsilon \neq 0$.

Suppose that $d T_{s}\left(h_{s}^{*}\right) \neq 0, s=1, \ldots, m$, and the non-degenerate case [10] is realized for the periodic solution. Then subsystems have only o-points, such that the MCCS is functioning in the main oscillations mode. Periodic motions of the generating MCCS constitute the family $\Sigma$, where the period depends on a single parameter, according to law [11-13].

We will later omit parameters $\gamma_{s}$ in order to focus on finding the vector $h^{*}$. 
Denote

$$
x\left(\varepsilon, x^{0}, t\right)=\left(x^{1}\left(\varepsilon, x^{0}, t\right), \ldots, x^{m}\left(\varepsilon, x^{0}, t\right)\right)
$$

the solution of (1) with the initial point $x^{0}$ (at $t=0$ ). Calculate the partial derivative of (3) with respect to $\varepsilon$ at $\varepsilon=0$ in the case when the solution of (3) coincides with that of (2). This derivative is the solution of the following linear system with zero initial conditions:

$$
\begin{array}{r}
\left.\frac{d}{d t}\left(\frac{\partial x_{k}^{s}}{\partial \varepsilon}\right)=\sum_{j=1}^{m_{s}} p_{k j}^{s}\left(h_{s}^{*}, t\right)\left(\frac{\partial x_{j}^{s}}{\partial \varepsilon}\right)+\tilde{X}_{k}^{s}\left(0, \varphi^{1}\left(h_{1}^{*}, t\right), \ldots, \varphi^{m}\left(h_{m}^{*}, t\right)\right)\right), \\
p_{k j}^{s}\left(h_{s}^{*}, t\right)=\left(\frac{\partial X_{k}^{s}}{\partial x_{j}^{s}}\right)_{*}, \quad s=1, \ldots, m, \quad k=1, \ldots, m_{s},
\end{array}
$$

where the symbol $*$ by the parentheses means that (2) with $h=h^{*}$ is substituted into the partial derivatives.

The homogeneous part of (4) decouples into $m$ subsystems, each containing its own parameter $h_{s}^{*}$ and admitting a single $T_{s}\left(h_{s}^{*}\right)$-periodic solution, where $T_{s}\left(h_{s}^{*}\right)=2 \pi$. The corresponding conjugated system decouples into $m$ subsystems too. Denote $\left\{\psi_{k}^{s}\left(h_{s}^{*}, t\right)\right\}$ $2 \pi$-periodic solutions of the conjugated system.

Now we can write conditions for $2 \pi$-periodic solutions of (4) to exist in the form

$$
g\left(h^{*}\right)=0,
$$

where $g(h)=\left\{g^{1}(h), \ldots, g^{m}(h)\right\}$,

$$
g^{s}(h) \equiv \int_{0}^{2 \pi} \sum_{k=1}^{m_{s}} \tilde{X}_{k}\left(0, \varphi^{1}\left(h_{1}, t\right), \ldots, \varphi^{m}\left(h_{m}, t\right)\right) \psi_{k}^{s}\left(h_{s}, t\right) d t, \quad s=1, \ldots, m .
$$

We call (5) the system of amplitude equations.

On the one hand, (5) gives necessary conditions for $2 \pi$-periodic solutions of (1). On the other hand, (5) represents a system of $m$ algebraic equations with respect to $h_{1}^{*}, \ldots, h_{m}^{*}$. If (5) is consistent for a given $h^{*}$, then the necessary conditions for (1) to have a periodic solution are satisfied. Moreover, (5) turns out to be sufficient in the case of simple roots.

Consider the concept of cycles:

Definition 2. An isolated periodic solution of an autonomous system is called cycle.

Theorem 1. Each simple root of amplitude equations (5) implies a unique cycle of (1).

Remark 1. Under conditions of Theorem 1 the family of periodic solutions $\Sigma$ bifurcates such that cycles emerge.

To prove Theorem 1 [10] we use the implicit function theorem, which is applied to find necessary and sufficient conditions of a periodic solution. Since subsystems have o-points, the system of $m$ equations that represents conditions of periodic solution coinsides with amplitude equations (5).

The following theorem is used to calculate the Lyapunov exponents (LE) of a cycle in MCCS: [10] 
Theorem 2. Given conditions of Theorem 1, the cycle has $m$ simple zero LEs, $m$ nonzero LEs of the form $\lambda=\alpha \varepsilon+o(\varepsilon), \alpha \neq 0$, where numbers $\alpha$ are defined by the algebraic equation

$$
\operatorname{det}\left\|\frac{\partial g^{s}\left(h^{*}\right)}{\partial h_{j}}-2 \pi \alpha \delta_{s j}\right\|=0
$$

and nonzero LEs inherited from the generating solution.

\section{Conclusion}

MCCS allows to take into account connections of systems. Among the characteristic features of MCCS there are: multi-level hierarchical structure, various modes of functioning, nonlinear models, high order. MCCS can also be classified as large-scale system. A natural approach to study MCCS proposed in [1] takes into account its structure and the dynamics of subsystems, and considers the connections between subsystems as "action is equal to reaction".

\section{References}

[1] V.N. TKhaI, Model with Coupled Subsystems, Autom. Remote Control. 2013. V. 74. No. 6. P. 919-931.

[2] V.M. Matrosov, Vector Lyapunov Functions Method to Analyse Complex Systems with Distributed Parameters, Autom. Remote Control. 1973. V. 34. No. 1. P. 5-22.

[3] V.M. Matrosov And A.A. Voronov, Eds., Vector Lyapunov Functions Method in Theory of Stability, Moscow: Nauka, 1987. (in Russian).

[4] V.M. Matrosov, Vector Lyapunov Functions Method: Analysis of Dynamics of Nonlinear Systems, Moscow: Fizmatlit, 2001. (in Russian).

[5] D.R. Merkin, Gyroscopic Systems, Moscow: Nauka, 1974. (in Russian)

[6] V.I. Zubov, Analytical Dynamics of a Set of Bodies, S-Petersburg: Leningrad State University, 1983. (in Russian).

[7] E.S. Pyatnitskiy, A Decoupling Principle in Control of Mechanical Systems, Reports of the Russian Academy of Sciences. 1988. Vol. 300. No. 2. P. 300303. (in Russian).

[8] D. SiljaK, Decentralized Control of Complex Systems, Boston: Academic press, 1991.

[9] F.L. Chernousko, I.M. Ananievskiy, S.A. Reshmin, Control Methods for Nonlinear Mechanical Systems, Moscow: Fizmatlit, 2006. (in Russian)

[10] V.N. TKhai, The Period on a Family of Nonlinear Oscillations and Periodic Motions of a Perturbed System at a Critical Point of the Family, J. Appl. Math. Mech. 2010. V. 74. No. 5. P. 579-586.

[11] V.N. TKhaI, The Law of the Dependence of the Period of Nonlinear Oscillations on a Single Parameter, J. Appl. Math. Mech. 2011. V. 73, No. 3, P. 304-307.

[12] V.N. TKHAI, The behaviour of the symmetrical periodic motions, J. Appl. Math. Mech. 2012. V. 76. No. 4. P. 610-615. 
[13] V.N. Tkhai. Oscillations in the Autonomous Model Containing Coupled Subsystems // Autom. Remote Control. 2014. V. 75 (in print).

[14] V.N.TKhaI, A Model Containing Coupled Subsystems. Cycles Case in the Subsystems, Autom. Remote Control. 2014. V. 75 (in print).

[15] V.N. TKHAI, A Mechanical System Containing Weakly Coupled Subsystems, J. Appl. Math. Mech. 2013. Vol. 77. No.6. 